\title{
SÍNTESIS DE HIDROGELES DE QUITOSANO A PARTIR DE CÁSCARA DE CAMARÓN PARA ENSAYOS DE ADSORCIÓN DE COBRE
}

\author{
Reyna Guadalupe SÁNCHEZ-DUARTE, María del Rosario MARTÍNEZ-MACÍAS, \\ Ma. Araceli CORREA-MURRIETA*, Jorge SALDÍVAR-CABRALES, \\ Dalia Isabel SÁNCHEZ-MACHADO y Jaime LÓPEZ-CERVANTES
}

Departamento de Ciencias del Agua y Medio Ambiente, Instituto Tecnológico de Sonora. Calle 5 de febrero 818 Sur, Ciudad Obregón, Sonora, México, C. P. 85100

*Autor para correspondencia: maria.correa@itson.edu.mx

(Recibido julio 2015; aceptado agosto 2016)

Palabras clave: aguas ácidas, metales pesados, bioadsorbente, remoción

\section{RESUMEN}

La minería representa una de las actividades económicas más importantes para muchos países y México no es la excepción. Sin embargo, los residuos líquidos que genera esta actividad suponen un riesgo para el ambiente debido a que se caracterizan por su elevado contenido de iones metálicos y valores bajos de $\mathrm{pH}$. Actualmente, la adsorción en quitosano constituye una alternativa a los tratamientos convencionales para la recuperación de metales pesados en solución acuosa. En este estudio se obtuvieron hidrogeles de quitosano puro y modificado a partir del exoesqueleto de camarón para llevar a cabo ensayos de adsorción de cobre en solución ácida, con particular referencia a los efectos del tiempo de contacto, $\mathrm{pH}$ de la solución, masa del adsorbente y concentración inicial de cobre. Los resultados experimentales mostraron que las condiciones óptimas de adsorción de cobre fueron a $\mathrm{pH} 4$, con $4.5 \mathrm{~g}$ de adsorbente (quitosano puro) y $4 \mathrm{~h}$ de tiempo de contacto, con estas condiciones se alcanzó una remoción del $99.35 \%$. Adicionalmente, se llevaron a cabo ensayos de adsorción con 160, 250 y 416 ppm de concentración de cobre en solución ácida y se observó que, a menor concentración mayor porcentaje de remoción, con hasta $92 \%$ de remoción para la concentración de 160 ppm, así como 72 y $67.3 \%$ para las concentraciones de 250 y 416 ppm, respectivamente. Lo anterior sugiere que el hidrogel de quitosano puro en forma de perlas, puede ser usado como adsorbente efectivo en la remoción de cobre presente en lixiviados de minas.

Key words: acidic waters, heavy metals, biosorbent, removal

\begin{abstract}
Mining is one of the most important economic activities for many countries and Mexico is not the exception. However, liquid wastes generated by this activity pose a risk to the environment because they are characterized by its high content of metal ions and low $\mathrm{pH}$ values. Currently, the adsorption process in chitosan is an alternative to conventional treatments in the recovery of heavy metals from aqueous solutions. In this study we obtained both, pure and modified chitosan hydrogels from shrimp shell to carry out adsorption tests of copper in acid solution. The effects of contact time, $\mathrm{pH}$,
\end{abstract}


adsorbent mass, and initial copper concentration were investigated. The experimental results showed that the optimal adsorption conditions of copper were as follows: $\mathrm{pH} 4$, adsorbent mass (pure chitosan) $4.5 \mathrm{~g}$, and contact time of $4 \mathrm{~h}$. Under these conditions, the $99.35 \%$ of removal was achieved. In addition, adsorption tests were conducted with three different initial concentrations (160,250 and $416 \mathrm{ppm})$ of copper in acid solution. It was observed that, at lower concentration the higher removal percentage, reaching up to $92 \%$ for $160 \mathrm{ppm}$, as well as 72 and $67.3 \%$ for 250 and $416 \mathrm{ppm}$, respectively. As a result, the pure chitosan hydrogel beads can be used as an effective adsorbent for the removal of copper from mining leachates.

\section{INTRODUCCIÓN}

En la actualidad, la contaminación de medios acuáticos por metales pesados es un serio problema ambiental, principalmente debido a las descargas de residuos industriales. Los metales pesados a diferencia de los contaminantes orgánicos no son biodegradables y se pueden acumular en tejidos vivos. La contaminación por aguas ácidas de drenaje que contienen metales pesados, es un problema común en muchas zonas metalogénicas y particularmente importante en explotaciones mineras de carbón o de metales. Los drenajes ácidos de antiguas explotaciones de carbón y minería metálica son las principales fuentes de contaminación de aguas subterráneas en el mundo, debido a que contienen una gran cantidad de sólidos en suspensión y un alto contenido de sulfato y metales disueltos (Fe, Al, Mn, $\mathrm{Zn}, \mathrm{Cu}, \mathrm{Pb}$, etc.). Se han alcanzado concentraciones de decenas y centenas de $\mathrm{mg} / \mathrm{L}$. Además, estos contaminantes por estar a esas concentraciones son nocivos para la actividad biológica, mortales para peces y crustáceos de río. Además, afectan al ganado y destruyen cultivos y riberas. Asimismo contaminan los cauces de ríos, cuyas aguas pueden llegar a dañar la estructura y cimentación de construcciones (García et al. 2003, Monier et al. 2010, Zhang 2011).

En los efluentes de minas se ha encontrado la presencia de cobre como sulfato de cobre, el cual proviene de los lavados efectuados en el proceso de electrodepositación. El cobre es un material ampliamente utilizado en la actualidad y es considerado como un elemento esencial para la vida humana. Sin embargo, y al igual que cualquier otro metal pesado, puede resultar potencialmente tóxico bajo ciertas concentraciones. Entre las distintas enfermedades asociadas a la presencia de cobre en el organismo humano se encuentran problemas de neurotoxicidad conocida como enfermedad de "Wilson", fallas de riñón y cáncer de pulmón (Agouborde 2008).

Las principales técnicas que han sido usadas para la reducción de concentraciones de metales pesados provenientes de residuos industriales son la precipitación química, intercambio iónico, filtración por membrana, métodos electrolíticos, ósmosis reversible y extracción con solventes (Xiong et al. 2009). No obstante, estos métodos son económicamente inviables por los altos costos de operación. Hoy en día se reconoce que la adsorción, usando adsorbentes de bajo costo, es un método eficaz y económico para la descontaminación de aguas. Especial atención se ha prestado a los polisacáridos tales como el quitosano, un aminopolímero natural que posee varias características intrínsecas que lo hace un bioadsorbente efectivo para la remoción de metales. El uso de este bioadsorbente es justificado porque es biodegradable y no tóxico (Díaz de Apodaca et al. 2007, Crini y Badot 2008, Liu et al. 2012).

El quitosano es un polisacárido lineal que se obtiene por desacetilación extensiva de la quitina y está compuesto por dos tipos de unidades estructurales distribuidas de manera aleatoria a lo largo de la cadena: la N-acetil-D-glucosamina y la D-glucosamina, las cuales se encuentran unidas entre sí por enlaces del tipo $\beta(1 \rightarrow 4)$ glicosídicos. La desacetilación de la quitina se lleva a cabo por hidrólisis de los grupos acetamida en medio fuertemente alcalino y a altas temperaturas. Generalmente la reacción se realiza en fase heterogénea con soluciones concentradas de $\mathrm{NaOH}$ o $\mathrm{KOH}(40-50 \%)$ a temperaturas superiores a $100{ }^{\circ} \mathrm{C}$.

El quitosano se caracteriza por contener un elevado número de grupos amino libres, los cuales son muy reactivos para la quelación de cationes metálicos a $\mathrm{pH}$ más o menos neutro (Díaz de Apodaca et al. 2007).

La adsorción en quitosano es una tecnología que constituye una alternativa a los tratamientos convencionales para la recuperación de los metales pesados en solución acuosa. El objetivo de este estudio fue obtener hidrogeles de quitosano y quitosano modificado a partir del exoesqueleto de camarón, para realizar ensayos de adsorción de cobre en solución ácida, con particular referencia a los efectos del tiempo de contacto, $\mathrm{pH}$ de la solución, masa del adsorbente y concentración inicial. 


\section{MATERIALES Y MÉTODOS}

Los reactivos empleados en esta investigación fueron grado reactivo y las soluciones fueron preparadas con agua desionizada. Los materiales de vidrio utilizados se lavaron con $\mathrm{HCl}$ concentrado y se enjuagaron con agua destilada.

\section{Producción de quitosano y lixiviado de cobre}

El lixiviado de cobre se obtuvo a partir de una columna de lixiviación estática, rellena con mineral de cobre. Para lixiviar el mineral se utilizó ácido sulfúrico con una concentración de $10 \mathrm{~g} / \mathrm{L}$. Posteriormente, el lixiviado obtenido se diluyó hasta una concentración de 416 ppm, dicha solución fue utilizada durante todos los ensayos de adsorción. Para la producción de quitosano no comercial obtenido a partir del exoesqueleto de camarón por método químico, se siguió el método utilizado en trabajos previos (Sánchez-Duarte et al. 2012), el que involucra tres pasos: desmineralización, desproteinización y desacetilación del exoesqueleto. Brevemente, en la desmineralización, el exoesqueleto de camarón se coloca en una solución de $\mathrm{HCl} 1 \mathrm{M}(1: 10 \mathrm{~m} / \mathrm{v})$ y se agita mecánicamente por $4 \mathrm{~h}$ a temperatura ambiente. En la desproteinización, el exoesqueleto de camarón se introduce en una solución de $\mathrm{NaOH} 4.5 \%(1: 15 \mathrm{~m} / \mathrm{v})$ y se agita mecánicamente a $65^{\circ} \mathrm{C}$ en baño de aceite por $4 \mathrm{~h}$. Finalmente, en la desacetilación, el exoesqueleto se agita mecánicamente en una solución de $\mathrm{NaOH} 45 \%$ a $110{ }^{\circ} \mathrm{C}$ en baño de aceite por $2 \mathrm{~h}$. Después de cada paso, las muestras son lavadas con agua hasta tornarse neutras. El quitosano obtenido se seca a temperatura ambiente y se almacena.

\section{Síntesis de hidrogeles de quitosano puro y entre- cruzado}

Para la preparación de los hidrogeles adsorbentes se siguió el método propuesto por Correa-Murrieta et al. (2012) y Sánchez-Duarte et al. (2012). Para la síntesis de perlas de quitosano entrecruzadas con tripolifosfato de sodio, se prepara una solución de quitosano al $2 \%(\mathrm{~m} / \mathrm{v})$ en ácido acético al $1.5 \%(\mathrm{v} / \mathrm{v})$. La solución de quitosano se gotea de una pipeta en una solución de tripolifosfato de sodio al $1 \%(\mathrm{~m} / \mathrm{v})$, formándose las perlas de quitosano entrecruzado con tripolifosfato (q-TPP). Después de reposar por $12 \mathrm{~h}$, las perlas resultantes se lavan con agua destilada y se almacenan. Para la obtención de quitosano puro (q-puro), la solución de quitosano se gotea en una solución de $\mathrm{NaOH}$ al $0.5 \mathrm{M}$, las perlas resultantes se dejan reposar $16 \mathrm{~h}$. Posteriormente, se lavan con agua destilada hasta alcanzar un $\mathrm{pH} 7$ y se almacenan.
Finalmente, para la síntesis de perlas de quitosano entrecruzado con glutaraldehído (q-GLU), las perlas de quitosano puro se colocan en una solución de glutaraldehído al $0.025 \mathrm{M}$ y se dejan reposar por 12 $\mathrm{h}$, se lavan con agua destilada y se almacenan.

Los hidrogeles de quitosano se caracterizaron en función de la humedad, diámetro y solubilidad. Se midió el diámetro de las perlas con un vernier digital Cole-Parmer (EW-97152-06) y se les determinó el \% de humedad con el método establecido por la AOAC (2005). Para determinar la solubilidad se colocó una masa conocida de adsorbente en ácido acético $5 \%$ $(\mathrm{v} / \mathrm{v})$ por $24 \mathrm{~h}$.

\section{Ensayos de adsorción}

En general, las condiciones óptimas de adsorción se obtuvieron con procedimientos estándar, un volumen fijo de lixiviado de cobre, con una cantidad conocida de adsorbente en condiciones controladas de tiempo de contacto, velocidad de agitación, temperatura y $\mathrm{pH}$. Todas las pruebas se llevaron a cabo mediante el método por lotes. La concentración de cobre en solución se determinó por la formación de un complejo amoniacal de color azul intenso, basándose en el método de Harris (2010), con modificaciones. La curva de calibración fue preparada a partir de soluciones de sulfato de cobre $(0$ a 620 ppm) y amoníaco en solución al $25 \%$, en la que se obtuvo un coeficiente de determinación $\mathrm{R}^{2}=0.995$. La concentración del metal se midió al poner en contacto $5 \mathrm{~mL}$ de lixiviado de cobre con $5 \mathrm{~mL}$ de amoníaco en solución al $25 \%$ y se aforó con agua desionizada en matraces de $25 \mathrm{~mL}$. Finalmente, se midió la absorbancia en un espectrofotómetro UV/ vis (Genesys $10 \mathrm{UV}$ scanning) a $610 \mathrm{~nm}$. Las condiciones óptimas de adsorción examinadas fueron el $\mathrm{pH}$ de la solución, el tiempo de equilibrio y la masa del adsorbente. Se estimaron los valores promedio y las desviaciones estándar.

Los $\mathrm{pH}$ estudiados fueron 3, 4, 5, 6 y 2.4 (pH propio del lixiviado de cobre). Estos $\mathrm{pH}$ fueron utilizados debido a que el cobre precipita a valores mayores de 6 unidades (Noguerol 1996). El pH se ajustó mediante la adición de $\mathrm{NaOH} 6 \mathrm{M}$ y se midió con un medidor digital (Hanna Instruments, Woonsocket, Rhode Island, USA). Una cantidad conocida de adsorbente (derivado de quitosano) se colocó en matraces con un mismo $\mathrm{pH}$ y volumen de solución de lixiviado, luego se agitó mecánicamente a $280 \mathrm{rpm}$ en un agitador Multi Vortex-Genie Digital (Scientific Industries, Inc., USA), por $60 \mathrm{~min}$ a temperatura ambiente, el $\mathrm{pH}$ y el adsorbente óptimo fueron aquellos en los que se obtuvo el porcentaje de remoción (\% Rem) más alto. 
Para determinar el tiempo de contacto óptimo, varios matraces con solución de lixiviado ajustada a su $\mathrm{pH}$ óptimo y $1 \mathrm{~g}$ de adsorbente se agitaron a intervalos de tiempo graduales. Por último, para determinar la cantidad óptima de adsorbente, diferentes cantidades de adsorbente (1, 2, 3, 4, 4.5 y $5 \mathrm{~g})$ se pusieron en contacto con la solución de lixiviado a su $\mathrm{pH}$, adsorbente y tiempo óptimos ya determinados. Todas las pruebas de adsorción se llevaron a cabo por duplicado. E1 \% Rem fue calculado con la siguiente ecuación:

$\%$ Rem $=\frac{\left(C_{o}-C_{f}\right)}{C_{o}} \times 100$

Donde $C_{o}$ y $C_{f}$ son la concentración inicial y final de la solución, respectivamente.

\section{RESULTADOS Y DISCUSIÓN}

Caracterización de los adsorbentes. El cuadro I, resume los resultados obtenidos para todos los adsorbentes sintetizados en cuanto a diámetro, \% de humedad y solubilidad. Los adsorbentes de quitosano entrecruzado (q-TPP, q-GLU) resultaron insolubles en medio ácido, en tanto que el de quitosano puro sí fue soluble en dicho medio. Resultados similares fueron obtenidos por Díaz de Apodaca et al. (2007) y Crini y Badot (2008). La propiedad ácido-base del quitosano conduce a la protonación de los grupos amino a pH ácidos, lo que provoca la disolución total o parcial del quitosano puro en soluciones ácidas (excepto en disoluciones de ácido sulfúrico).

CUADRO I. CARACTERÍSTICAS DE HIDROGELES DE QUITOSANO

\begin{tabular}{llcl}
\hline Adsorbente & Solubilidad* & Diámetro $(\mathrm{mm})$ & Humedad (\%) \\
\hline q- puro & Soluble & $4.06 \pm 0.21$ & $97.23 \pm 0.012$ \\
q-TPP & Insoluble & $3.11 \pm 0.11$ & $93.17 \pm 0.015$ \\
q-GLU & Insoluble & $4.13 \pm 0.17$ & $94.70 \pm 0.15$ \\
\hline
\end{tabular}

* solución de ácido acético 5 \% (v/v), q-puro = quitosano-puro, q-TPP = quitosano-tripolifosfato, q-GLU = quitosano-glutaraldehído. Media \pm desviación estándar, $\mathrm{n}=50$ para diámetro, $\mathrm{n}$ $=3$ para humedad

Ensayos de adsorción. La concentración de cobre en el lixiviado con la que se trabajó durante todos los ensayos de adsorción fue de 416 ppm.

Efecto del pH. En la figura 1 se observa que el $\mathrm{pH}$ óptimo de adsorción fue de 4 en todos los adsorbentes, dicho resultado es similar al obtenido por Juang y Shao (2002) y Benavente et al. (2011), quienes mencionan que en los experimentos de adsorción con cationes metálicos, el \% Rem depende en gran medida del $\mathrm{pH}$ de la solución. Esto se debe a que a $\mathrm{pH}$ bajo tiende a disminuir el número de sitios de unión para iones metálicos, es decir, en metales catiónicos a mayor $\mathrm{pH}$ se tiene el mayor $\%$ Rem. Adicionalmente, se probó con $\mathrm{pH} 5$ y 6 , en los que se observó una precipitación total del cobre presente en la solución. El desarrollo de estos ensayos permitió al mismo tiempo la selección del adsorbente óptimo, en este caso fue el quitosano puro. La adsorción de cobre a $\mathrm{pH} 4$ fue mayor en q-puro, seguido de q-TPP y q-GLU con un $\%$ Rem de $15.91,12.81$ y 7.76 , respectivamente. Para estos ensayos se utilizó $1 \mathrm{~g}$ de adsorbente, $50 \mathrm{~mL}$ de lixiviado de cobre y los $\mathrm{pH}$ fueron ajustados con $\mathrm{HCl}$ o $\mathrm{NaOH}$ según cada caso.

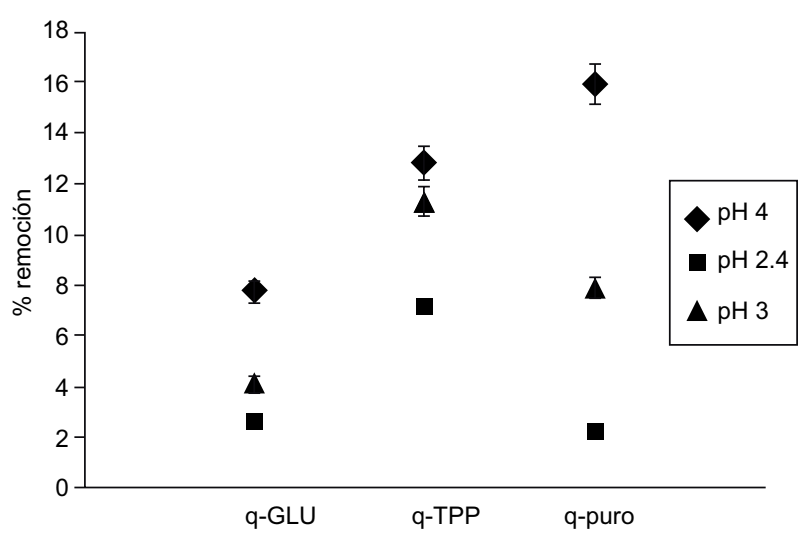

Fig. 1. Efecto del pH en la adsorción de cobre (masa de adsorbente $=1 \mathrm{~g}$, tiempo de contacto $=1 \mathrm{~h}, \mathrm{q}$-puro $=$ quitosano-puro, q-TPP = quitosano-tripolifosfato, q-GLU = quitosano-glutaraldehído. Media \pm desviación estándar, $\mathrm{n}=2$

Cantidad de adsorbente. Crini y Badot (2008) mencionan que además de factores como el $\mathrm{pH}$, la cantidad de adsorbente es importante porque determina el grado de adsorción y también puede ser usado para predecir el costo de quitosano por unidad de solución a ser tratada. La figura 2 muestra el efecto de la cantidad de q-puro usado en la adsorción de cobre a un tiempo de 60 min y $50 \mathrm{~mL}$ de solución de lixiviado con una concentración de cobre de 416 ppm. E1 \% Rem aumenta con la cantidad de adsorbente y permanece más o menos constante a partir de $4.5 \mathrm{~g}$ de perlas húmedas de q-puro. Lo anterior se debe a que a mayor cantidad de adsorbente, existen más sitios disponibles de adsorción para remover mayor 
cantidad de iones de cobre. En este trabajo se obtuvo que la masa óptima de adsorbente para cobre fue de $4.5 \mathrm{~g}$ de perlas húmedas de q-puro.

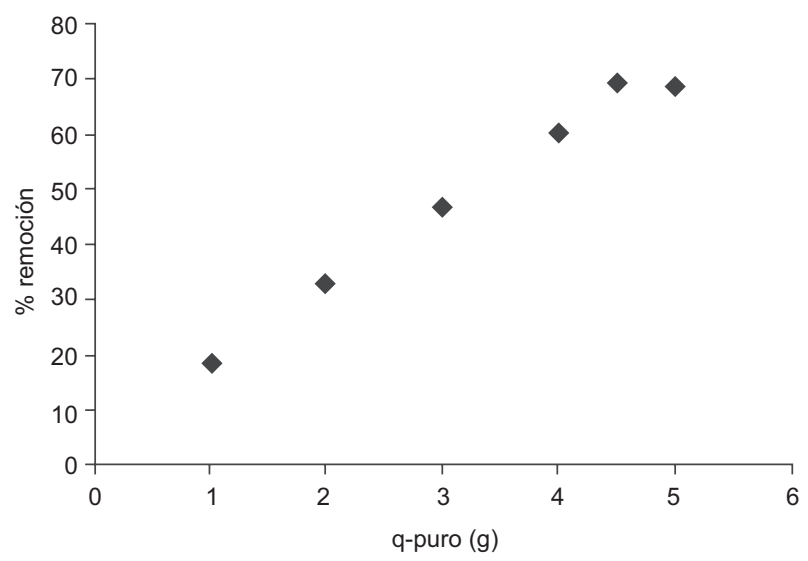

Fig. 2. Efecto de la cantidad de masa de quitosano puro (q-puro) en la adsorción de cobre (lixiviado de cobre a $\mathrm{pH} 4$, tiempo de contacto $=1 \mathrm{~h}$ )

Efecto del tiempo de contacto. La figura 3 muestra el efecto del tiempo de contacto sobre la adsorción de cobre con $4.5 \mathrm{~g}$ de perlas de q-puro y $50 \mathrm{~mL}$ de solución de lixiviado a $\mathrm{pH} \mathrm{4,} \mathrm{mostrando}$ que la adsorción de cobre aumenta con el incremento en el tiempo de contacto. Durante la primer hora se alcanza $67.3 \%$ de Rem y al cabo de 4 h, $99.35 \%$, manteniéndose más o menos constante en este porcentaje., Según Kumar et al. (2010), lo anterior pudo deberse a que durante el proceso, la superficie adsorbente se ocupa progresivamente por los iones metálicos hasta que alcanza un valor constante de adsorción que indica que ésta ha sido saturada. Por lo que el tiempo óptimo de adsorción alcanzado en

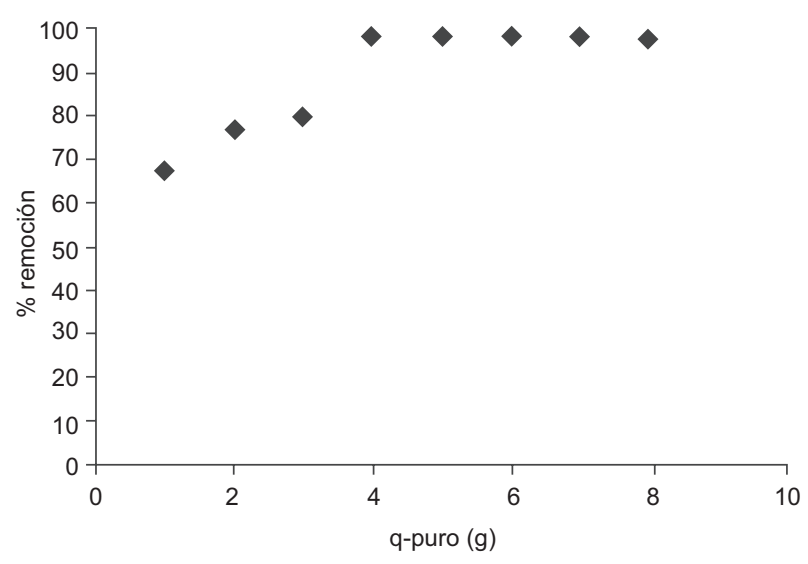

Fig. 3. Efecto del tiempo de contacto en la adsorción de cobre (masa de adsorbente $=4.5 \mathrm{~g}$, lixiviado de cobre a $\mathrm{pH} 4$ ) este ensayo se consideró de $4 \mathrm{~h}$, debido a que la mayor parte de la remoción del metal fue obtenida en este tiempo.

Efecto de la concentración inicial. Se pudo observar que a menor concentración de cobre en solución, mayor porcentaje de remoción. Se alcanzó hasta $92 \%$ de remoción para la concentración de 160 ppm, y de 72 y $67.3 \%$ para la concentración de 250 y 416 ppm, respectivamente. Esto indica que la concentración inicial juega un papel importante en la adsorción. Aunque, es importante mencionar que se deben ajustar las cantidades de adsorbente para cada concentración. Resultados similares fueron observados por Kumar et al. (2009).

\section{CONCLUSIÓN}

Se llevaron a cabo estudios de adsorción de cobre por hidrogeles de quitosano a diferentes valores de $\mathrm{pH}$ y condiciones experimentales en lote. Los resultados indican que la adsorción de cobre es dependiente del pH y de la concentración inicial de contaminante. Asimismo, se obtuvieron condiciones óptimas de tratamiento de $4 \mathrm{~h}, \mathrm{pH} 4$ y $4.5 \mathrm{~g}$ de adsorbente (q-puro). El hidrogel de q-puro en forma de perlas puede ser usado como un adsorbente efectivo en la remoción de cobre presente en lixiviados de minas.

\section{AGRADECIMIENTOS}

Esta investigación fue financiada por Programa de Fomento y Apoyo a Proyectos de Investigación (PROFAPI) 2014-1371.

\section{REFERENCIAS}

Agouborde M. L. I. (2008). Remoción de metales pesados por medio de adsorbentes no convencionales. Tesis de Maestría. Universidad de la Frontera. Temuco, Chile, $125 \mathrm{pp}$.

AOAC (2005). Method 925.09. Official methods of analysis of AOAC international. $18^{\text {th }}$ edition. Association of Official Analytical Chemist. Gaithersburg, Maryland, EUA, [en línea] http://www.eoma.aoac.org/ 24/08/16

Benavente M., Moreno L. y Martínez J. (2011). Sorption of heavy metals from gold mining wastewater using chitosan. J. Taiwan Inst. Chem. E. 42 (6), 976-988. DOI: $10.1016 /$ j.jtice.2011.05.003

Correa-Murrieta M. A., López-Cervantes J., SánchezMachado D. I., Sánchez-Duarte R. G., Rodríguez- 
Núñez J. R. y Núñez-Gastélum J. A. (2012). Fe(II) and $\mathrm{Fe}$ (III) adsorption by chitosan-tripolyphosphate beads: kinetic and equilibrium studies. J. Water Supply: Research and Technol-AQUA 61 (6), 331-341. DOI: 10.2166/aqua.2012.048

Crini G. y Badot P. M. (2008). Application of chitosan, a natural aminopolysaccharide, for dye removal from aqueous solutions by adsorption processes using batch studies: A review of recent literature. Prog. Polym. Sci. 33 (4), 399-447.

DOI: $10.1016 /$ j.progpolymsci.2007.11.001

Díaz de Apodaca D. E., Villarán-Velasco M. C., RíoPérez F., Ramírez-López C. A. e Ibarreta L. L. (2007). Utilización de adsorbentes basados en quitosano y alginato sódico para la eliminación de iones metálicos: $\mathrm{Cu} 2+, \mathrm{Pb} 2+, \mathrm{Cr} 3+$ y $\mathrm{Co} 2+$. Revista Iberoamericana de Polímeros 8 (1), 20-37.

García R., Ramírez P. y Manzano M. (2003). Bioadsorción de metales pesados de aguas ácidas de minas (I). Revista Ingeniería Química 401, 155-168.

Harris D. C. (2010). Quantitative chemical analysis. 8a ed. W. H. Freeman and Company. Nueva York, EUA, 719 pp.

Juang R. S. y Shao H. J. (2002). Effect of pH on competitive adsorption of $\mathrm{Cu}(\mathrm{II}), \mathrm{Ni}(\mathrm{II})$, and $\mathrm{Zn}$ (II) from water onto chitosan beads. Adsorption 8 (1), 71-78.

DOI: 10.1023/A:1015222607996

Kumar M., Tripathi B. P. y Shahi V. K. (2009). Crosslinked chitosan/polyvinyl alcohol blend beads for removal and recovery of Cd (II) from wastewater. J. Hazard. Mater. 172 (2-3), 1041-1048.

DOI: $10.1016 /$ j.jhazmat.2009.07.108
Kumar S., Ramakrishnan P., Dinesh K. S. y Sivanesan S. (2010). Thermodynamic and kinetic studies ofcadmium adsorption from aqueous solution onto rice husk. Braz. J. Chem. Eng. 27 (2), 347-355. DOI: 10.1590/S0104-66322010000200013

Liu Y., Kang Y., Huang D. y Wang A. (2012). $\mathrm{Cu}^{+2}$ removal from aqueous solution by modified chitosan hydrogels. J. Chem. Technol. Biotechnol. 87 (7), 1010-1016. DOI: $10.1002 /$ jctb. 3722

Monier M., Ayad D. M., Wei Y. y Sarhan A. A. (2010). Adsorption of $\mathrm{Cu}(\mathrm{II}), \mathrm{Co}(\mathrm{II})$, ad $\mathrm{Ni}$ (II) ions by modified magnetic chitosan chelating resin. J. Hazard. Mater. 177 (1-3), 962-970. DOI: 10.1016/j.jhazmat.2010.01.012

Noguerol J. A. (1996). Tecnologías limpias para la reducción de contaminantes y la recuperación de solutos de fuentes hidrominerales mediante resinas de intercambio iónico. Tesis Doctoral. Universitat Autónoma de Barcelona, Departamento de Química. Bellaterra, Barcelona, España, 133 pp.

Sánchez-Duarte R. G., Sánchez-Machado D. I., LópezCervantes J. y Correa-Murrieta M. A. (2012). Adsorption of allura red dye by cross-linked chitosan from shrimp waste. Water Sci. Technol. 65 (4), 618-623. DOI: 10.2166/wst.2012.900

Xiong C., Yao C., Wang L. y Ke J. (2009). Adsorption behavior of Cd (II) from aqueous solutions onto geltype weak acid resin. J. Hydromet. 98 (3-4), 318-324. DOI: 10.1016/j.hydromet.2009.05.008

Zhang M. (2011). Adsorption study of $\mathrm{Pb}(\mathrm{II}), \mathrm{Cu}(\mathrm{II})$ and $\mathrm{Zn}$ (II) from simulated acid mine drainage using dairy manure compost. Chem. Eng. J. 172 (1), 361-368. DOI: 10.1016/j.cej.2011.06.017 